Revue des patrimoines

37 | 2018

Jardins collectifs : de l'abbé Lemire aux jardins d'insertion. Typologies - Expériences - Enjeux de conservation

\title{
Les 20 printemps des jardins partagés français. Bilan et perspectives
}

Twenty years of community gardens in France, evaluation and perspectives

\section{Laurence Baudelet-Stelmacher}

\section{OpenEdition}

Journals

Édition électronique

URL : http://journals.openedition.org/insitu/19725

DOI : 10.4000/insitu. 19725

ISSN : $1630-7305$

Éditeur

Ministère de la culture

Référence électronique

Laurence Baudelet-Stelmacher, « Les 20 printemps des jardins partagés français. Bilan et

perspectives », In Situ [En ligne], 37 | 2018, mis en ligne le 19 décembre 2018, consulté le 19 avril 2019. URL : http://journals.openedition.org/insitu/19725 ; DOI : 10.4000/insitu.19725

Ce document a été généré automatiquement le 19 avril 2019

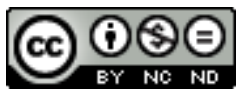

In Situ Revues des patrimoines est mis à disposition selon les termes de la licence Creative Commons Attribution - Pas d'Utilisation Commerciale - Pas de Modification 4.0 International. 


\title{
Les 20 printemps des jardins partagés français. Bilan et perspectives
}

Twenty years of community gardens in France, evaluation and perspectives

\author{
Laurence Baudelet-Stelmacher
}

\begin{abstract}
"Un mode nouveau de récit historique apparaît, disons "le récitatif" de la conjoncture, du cycle, voire de "l'intercycle" qui propose à notre choix une dizaine d'années, un quart de siècle et à l'extrême limite, le demi-siècle du cycle classique de Kondratieff $»^{1}$.

Fernand Braudel
\end{abstract}

1 Cet article porte un regard rétrospectif sur les deux premières décennies des jardins partagés, un mouvement de jardinage associatif qui s'est constitué en 1997. Vingt années se sont écoulées, ce qui est peu mais constitue néanmoins un intervalle de temps suffisant pour la production d'un récit historique. Fernand Braudel l'a appelé le « récitatif » d'une conjoncture économique ou sociale dans son article de 1958, distinguant différentes durées en histoire et dans les sciences humaines. Nous essaierons dans un premier temps de qualifier la «conjoncture » dans laquelle sont nés les jardins partagés, et à laquelle nous appartenons encore, selon nous.

2 Nous replacerons ces jardins dans le paysage du jardinage associatif en France qui s'est fortement diversifié et «hybridé» depuis les années 1990 tant dans les pratiques que dans le lexique. Nous en esquisserons une typologie simplifiée.

3 Notre analyse s'appuie sur une connaissance militante et réflexive. Nous avons participé directement à la naissance de ce mouvement en 1997 et pris part activement à son développement en Île-de-France en cofondant l'association Graine de Jardins en 2001 pour laquelle nous travaillons aujourd'hui. En parallèle, au cours de ces vingt années, nous avons eu le bonheur de dialoguer régulièrement avec des chercheurs en sciences sociales sur l'évolution des jardins partagés. Cela nous a permis d'utiliser les outils de l'ethnologie, notre formation initiale, pour prendre du recul et essayer de comprendre ce qui se jouait. 


\section{La naissance du mouvement des jardins partagés}

Le mouvement des jardins partagés émerge en 1997 avec la rédaction de la charte du Jardin dans tous états - La terre ${ }^{2}$ en partage et la constitution du réseau du Jardin dans tous ses états, appuyée par la Fondation de France. L'année précédente, cette fondation avait commencé à réunir dans ses locaux parisiens un groupe informel d'acteurs du jardinage collectif et de l'écologie, venant de toute la France.

5 La particularité de ce groupe tient au fait qu'il est composé d'associations « historiques » représentant les jardins familiaux (Fédération nationale des jardins familiaux et collectifs, Jardinot ${ }^{3}$, Jardiniers de France ${ }^{4}$ ) et de «jeunes » structures qui agissent dans des domaines variés : l'insertion et la lutte contre les exclusions (les Jardins d'aujourd'hui ${ }^{5}$ à Bordeaux, les jardins Cultivons la Solidarité - embryon du réseau Cocagne ${ }^{6}$ - à Besançon, les initiateurs du Pades ${ }^{7}$ ); l'écologie urbaine (Chantier Nature à Lille); l'éducation à l'environnement (le réseau national École et Natures) et la diffusion des techniques de jardinage écologique (l'éditeur Terre Vivantes, qui publie la revue Les Quatre Saisons du jardinage). Leur diversité est représentative d'époques et de courants qui ont été favorables au jardinage collectif depuis le XIX ${ }^{e}$ siècle. Les jardins partagés s'inscrivent dans cette histoire, ils en sont le dernier avatar né au tournant du XxI ${ }^{\mathrm{e}}$ siècle.

6 La Fédération nationale des jardins familiaux et collectifs ${ }^{10}$ poursuit l'action de la Ligue $\mathrm{du}$ coin de terre et du foyer, fondée en 1896 par l'abbé Lemire. Les Jardiniers de France sont apparus vingt ans plus tôt, en 1876, à Valenciennes (Nord). La première vague de création de jardins appelés ouvriers ${ }^{11}$ est soutenue par les tenants du catholicisme social, de la philanthropie patronale et du mouvement hygiéniste ${ }^{12}$. Ils visent à améliorer les conditions de vie de la classe ouvrière tout en exerçant sur elle une forme de contrôle social. Les jardiniers s'emploieront à les contourner avec une grande ingéniosité ${ }^{13}$. En 1952, les jardins ouvriers sont rebaptisés jardins familiaux par la loi.

7 Le Jardin du cheminot dépose ses statuts en 1942, au cours de la Deuxième Guerre mondiale. Le gouvernement de Vichy encourage alors la création de nouveaux lotissements de jardins ouvriers pour pallier les problèmes d'approvisionnement et de sous-alimentation. Leur nombre va tripler de 1939 à $1943^{14}$. Les «Victory Gardens ", en Angleterre ou aux États-Unis, jouent le même rôle pendant les deux guerres. Les récoltes de ces jardins permettent de suppléer en partie la production agricole manquante. En 1943, les jardins ouvriers fournissent $3 \%$ des besoins en calories de la population française ${ }^{15}$. Plus près de nous, au début des années 1990, les datchas, les lopins de terre alloués aux ouvriers et employés durant la période soviétique, ont permis à beaucoup de personnes de subvenir à leurs besoins alimentaires au moment de l'effondrement de l'URSS ${ }^{16}$ et au-delà.

8 Nous arrivons aux années 1980 et au mouvement écologiste. Le réseau d'éducation à l'environnement, École et Nature ${ }^{17}$ (1983) et la revue Les Quatre Saisons du jardin bio ${ }^{18}$ (1980) sont issus de la prise de conscience qui s'est opérée dans les années 1970. Désormais, la disparition d'écosystèmes et d'espèces observée à l'échelle planétaire est corrélée à des activités humaines. L'éducation à l'environnement, nouveau développement de l'éducation populaire, cherche à sensibiliser le grand public à ces enjeux et le jardin est un bon support d'observation du vivant. 
9 Mais la réalité est tout autre sur le terrain. Les pratiques des jardiniers amateurs sont alors considérées comme particulièrement polluantes par le ministère de l'Environnement qui disposait de plusieurs études sur le sujet. L'emploi important de produits phytosanitaires et d'engrais de synthèse dans les jardins pour lutter contre les nuisibles et doper les récoltes se trouve particulièrement pointé du doigt au titre de la préservation de la biodiversité et de la qualité des eaux. La diffusion de pratiques culturales proposant des alternatives à ces produits est encore minoritaire et confidentielle en 1997. Nous pouvons mesurer le chemin parcouru jusqu'au vote de la loi portée par le sénateur Joël Labbé ${ }^{19}$ en 2014 et qui aboutira à l'interdiction, en 2019, de la vente de produits phytosanitaires aux jardiniers amateurs.

La charte du Jardin dans tous ses états prône « le respect de l'environnement par la mise en œuvre de modes de gestion et de pratiques culturales favorisant la biodiversité (sauvage ou domestique), respectueuses du vivant, intégrant une gestion écologique des cycles naturels, de l'eau et des déchets $»^{20}$ (fig. 1). Les jardins partagés vont contribuer à ce changement de paradigme. De nombreux jardiniers néophytes s'y initient à la lutte biologique contre les nuisibles, à la fabrication du compost ou à l'utilisation de préparations naturelles (le fameux purin d'ortie par exemple). Ce sera un point de clivage avec les associations de jardinage familial qui prendront véritablement le tournant sous l'impulsion du premier plan Écophyto ${ }^{21}$ (2008-2018) lancé dans la foulée du Grenelle de l'Environnement ${ }^{22}$. Mais n'idéalisons pas les jardins partagés, les conflits entre les jardiniers tueurs de limaces à coup de granulés bleus et ceux qui les piègent avec de la bière et de la cendre ont bien existé.

\section{Figure 1}

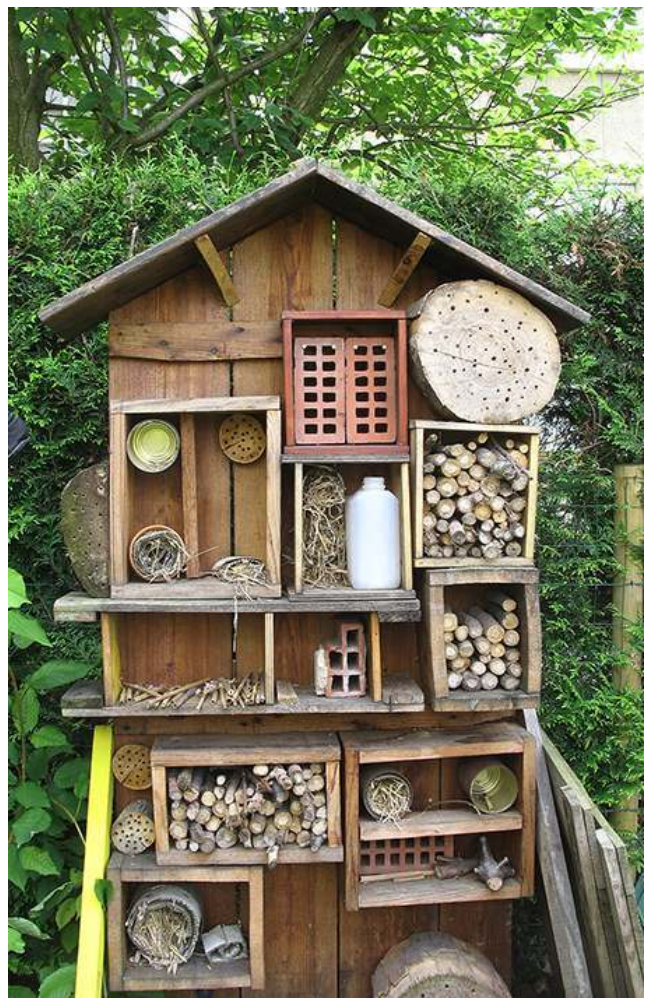

Hôtel à insectes - Jardin des Fabriques à Aubervilliers (Seine-Saint-Denis).

(c) Graine de Jardins, 2013. 


\section{L'influence du modèle nord-américain ${ }^{23}$}

11 Les jardins «communautaires" canadiens et américains vont influencer considérablement les promoteurs des jardins partagés français. Selon l'ethnologue Sandrine Baudry, « le terme américain de community, qu'il soit utilisé comme adjectif ou comme substantif, évoque l'inclusion et ses corollaires, la solidarité, le sentiment de sécurité, d'appartenance $»^{24}$. Nous sommes loin du caractère polémique que le mot « communautaire » revêt en français. L'appellation de « jardin communautaire » sera tout de même utilisée pendant plusieurs années par l'association lilloise des AJOnc ${ }^{25}$ avant d'être abandonnée. L'expression est également en usage au Canada francophone.

12 Les «community gardens" connaissent un regain d'intérêt dans les années 1970 à New York et dans plusieurs grandes villes des États-Unis, à un moment où les classes moyennes ont déserté les centres-villes pour aller vivre dans le pavillonnaire périurbain et où le mouvement hippie prône le «flower power ». Les immeubles vides laissent peu à peu place à des terrains en friche qui vont être transformés en jardins collectifs de quartier par les habitants appuyés par des associations comme «Green Guerillas ${ }^{26}$ ». Ils y cultivent toutes sortes de plantes alimentaires et ornementales, sans obligation de privilégier les premières sur les secondes. La dimension vivrière varie fortement d'un jardin à l'autre. Surtout, ils sont ouverts aux habitants du quartier selon des horaires affichés, il est possible d'y entrer sans être membre et d'y rester tant qu'un adhérent est présent. Ces petites oasis de verdure offrent des espaces de jeux pour les enfants dans des quartiers qui en sont dépourvus. À quoi s'ajoute un riche programme d'animations socioculturelles (repas de quartier, trocs, concerts, expositions, projections en plein air...) et d'ateliers pratiques qui permettent de diffuser des techniques adaptées aux contraintes urbaines comme le jardinage en carré27 ou les cultures en lasagne ${ }^{28}$.

13 L'implantation des "community gardens" dans les dents creuses de la ville les relie à l'habitat (fig. 2). Ils sont accessibles à pied ou en transports en commun et occupent pour certains de petites surfaces d'à peine quelques centaines de mètres carrés, l'équivalent de quatre à cinq parcelles individuelles de jardinage familial. À la même période en France, beaucoup de groupes de jardins familiaux se trouvent en périphérie, là où le foncier est disponible. 


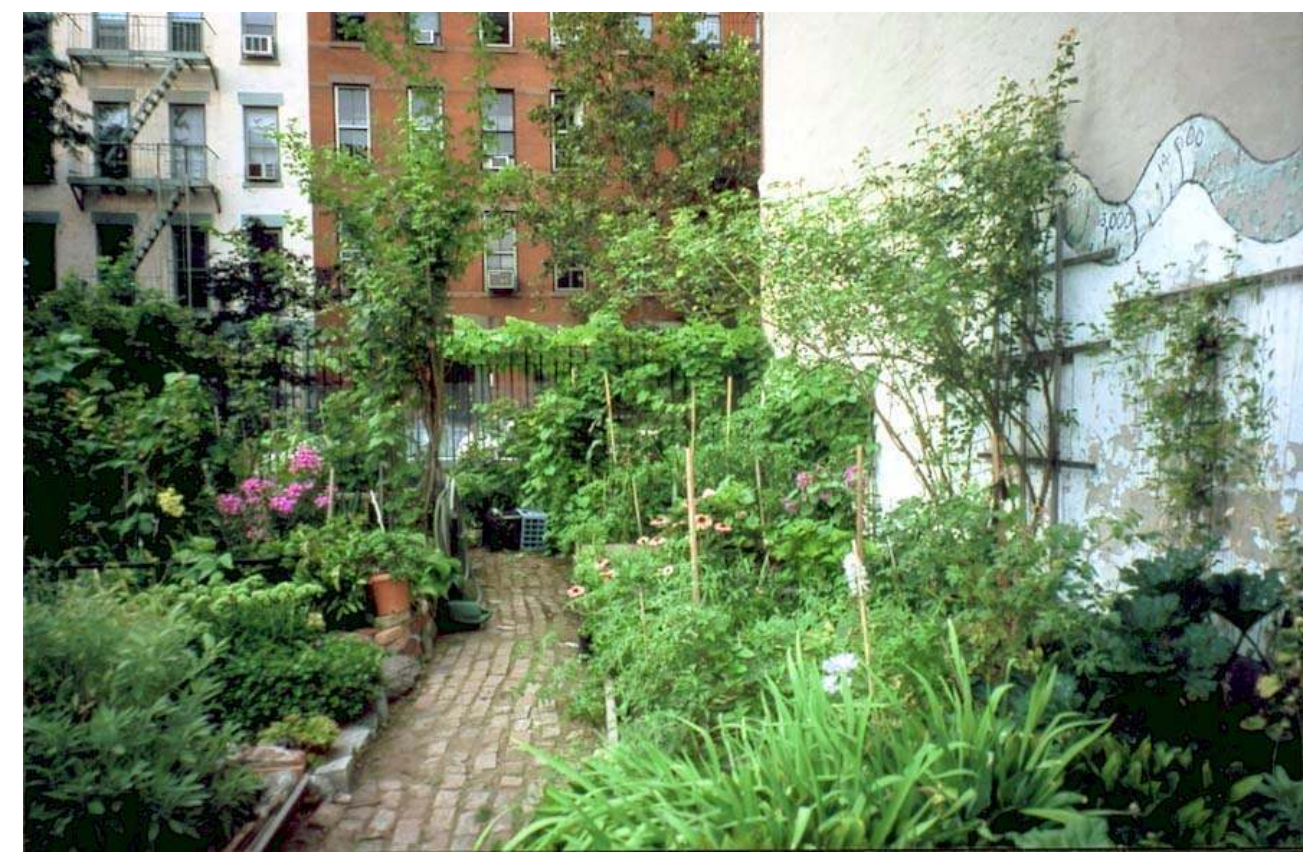

Clinton Community garden, 434 ouest 48e rue, New York (États-Unis), 2002.

(c) Laurence Baudelet.

14 Tout cela ne manque pas d'étonner les associations françaises participant à la conférence annuelle des "community gardens" nord-américains à Québec, en 1996, et qui visitent New York par la suite. Financés par la Fondation de France, ces voyages d'étude rassemblent les fondateurs du mouvement des jardins partagés. Ils y découvrent aussi les politiques publiques mises en œuvre pour soutenir ces initiatives citoyennes comme Green Thumb $^{29}$, à New York, qui a débuté en 1978 et a permis de régulariser l'accès au foncier municipal grâce à des conventions d'occupation des terrains.

Les échanges vont se développer durant toute la décennie suivante entre les acteurs des jardins partagés français et leurs homologues nord-américains, invités à témoigner de leur expérience lors des forums organisés par le Jardin dans tous ses états dans différentes villes.

16 Leur soutien donne une forte crédibilité au mouvement naissant et inspire aussi bien des projets de terrain que des politiques publiques comme le programme "Main Verte » à Paris.

\section{Les jardins partagés, un outil polyvalent de réponse aux crises}

17 La création du Jardin dans tous ses états témoigne d'une nouvelle dynamique en matière de jardinage collectif à un moment, la fin des années 1990, où la société française se trouve confrontée à de nouveaux défis, environnementaux mais aussi sociaux.

«La nouvelle pauvreté3 ${ }^{30}$ f fait son apparition dans le champ politique. Le chômage de masse, devenu structurel, se traduit par de l'exclusion sociale et l'isolement de publics découvrant la précarité. Les premières émeutes urbaines se produisent dans les banlieues 
pauvres de Lyon ou de Paris, révélant de profondes fractures au sein de la société française et appelant des réponses rapides de la part des pouvoirs publics. La création du ministère de la Politique de la Ville, en $1990^{31}$, survient dans ce contexte. La société civile n'est pas en reste. Les Restos du cœur apparaissent en 1985. La fin des années 1980 et le début des années 1990 voient aussi naître les jardins d'insertion sociale à Bordeaux avec les Jardins d'aujourd'hui (1988) et les chantiers d'insertion par l'activité économique en maraîchage avec les Jardins du cœur en 1989 et les jardins Cultivons la Solidarité en 1991 à Besançon, dont la production, cultivée en bio, est distribuée sous forme de paniers hebdomadaires à des adhérents.

19 À ces problématiques s'ajoutent de nouvelles attentes dans le champ politique. L'aspiration à une "démocratie participative » se fait jour, plaçant la question de la citoyenneté au centre des débats. Plus question de concevoir des jardins sans y associer les futurs jardiniers, ce qui n'était pas une démarche courante en 1997. Mais plus encore, l'objectif est d'encourager les projets portés et gérés par des habitants. La charte de La terre en partage promeut la concertation et la possibilité pour les citoyens de se réapproprier leur cadre de vie. L'association lyonnaise du Passe-jardins définit d'ailleurs les jardins partagés comme des « lieux de convivialité enracinés dans le territoire parce que conçus, créés et cultivés collectivement par les habitants $»^{32}$.

La perspective s'inverse, elle devient ascendante, de la base vers le sommet (bottom-up selon la terminologie anglophone). Et les modalités de gestion du projet, de " gouvernance » dirait-on aujourd'hui, font également l'objet d'une attention nouvelle. La charte de La terre en partage promeut une "gestion participative » et une élaboration collective des règles de fonctionnement là où s'appliquent majoritairement des règlements intérieurs types.

21 Mais la revendication première de ce jeune mouvement, c'est la restauration des liens sociaux (fig. 3). Le jardinage apparait comme un outil permettant de réunir des personnes appartenant à des générations, des classes sociales et des cultures différentes. C'est cette définition que va retenir la proposition de loi relative aux jardins collectifs votée par le Sénat en $2003^{33}$, à laquelle ont contribué les membres du Jardin dans tous ses états : « On entend par jardins partagés les jardins créés ou animés collectivement, ayant pour objet de développer des liens sociaux de proximité par le biais d'activités sociales, culturelles ou éducatives et étant accessibles au public ». 
Figure 3

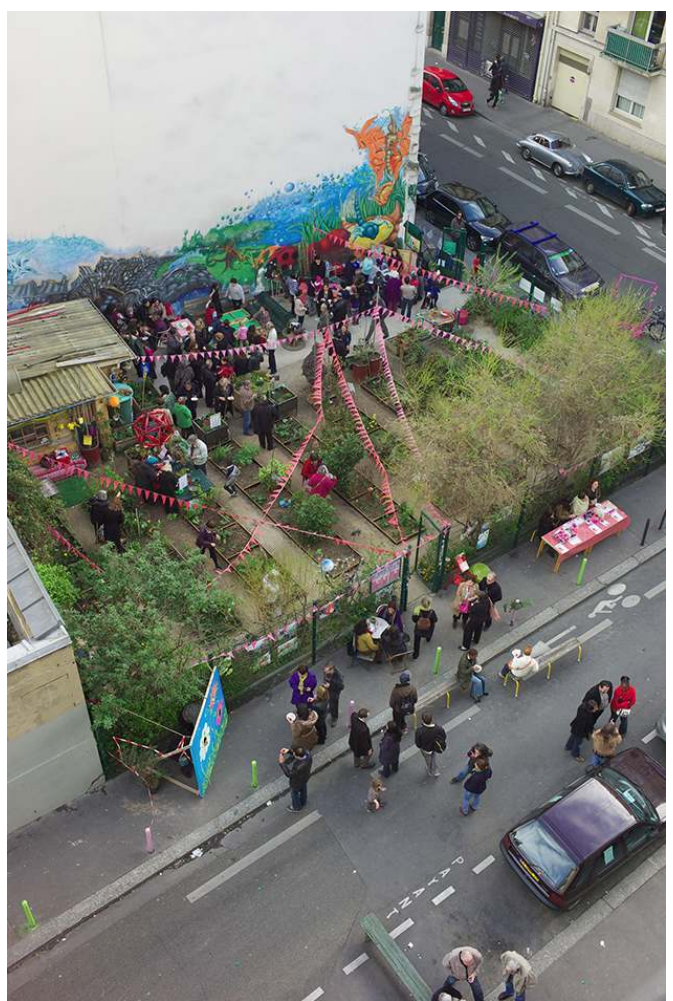

Jardin Nomade, Paris $11^{\mathrm{e}}$

(c) Pierre-Emmanuel WECK, 2013.

Dans cette même année 1997 se tient à Lille « le premier forum Jardinage et citoyenneté : le jardin dans tous ses états ", organisé par le Jardin dans tous ses états avec le soutien de la Fondation de France. Des acteurs associatifs nationaux, européens et nord-américains, et institutionnels s'y retrouvent et visitent le premier jardin " communautaire », le jardin des (Re)trouvailles situé dans le quartier Moulins (fig. 4). Celui-ci a été créé par l'association AJOnc qui développe ces jardins dans l'agglomération lilloise et bien au-delà. 


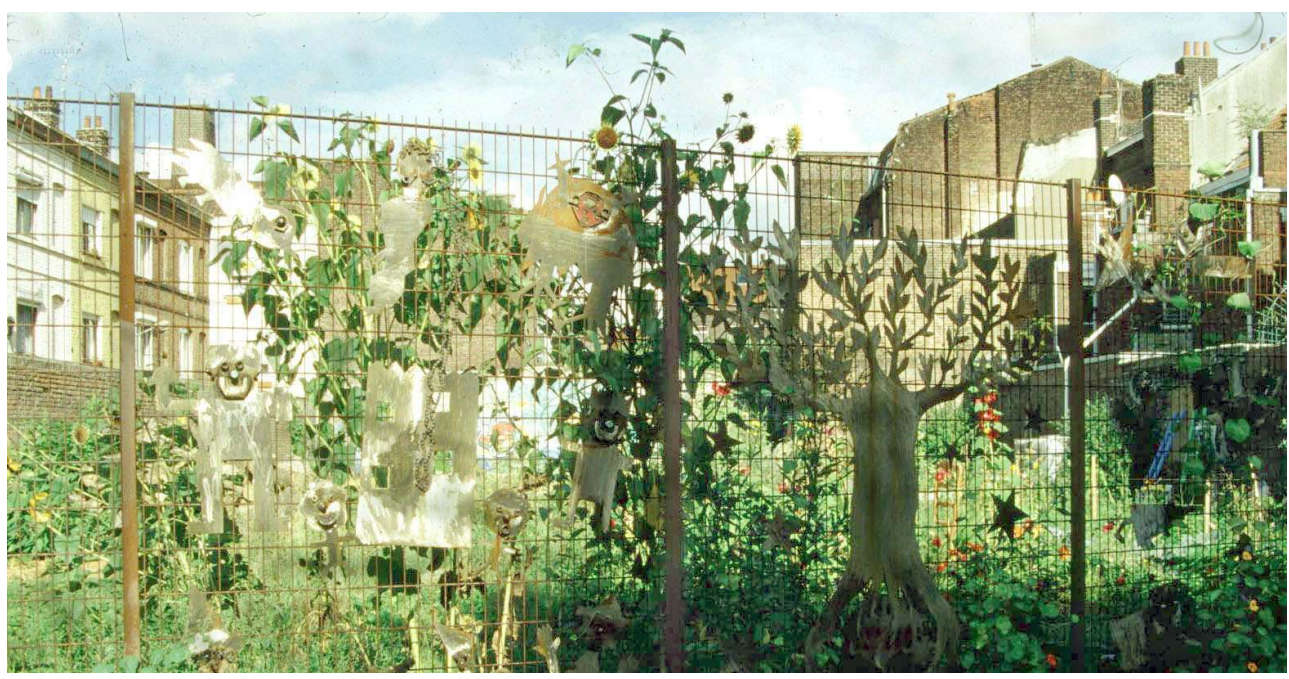

Clôture ouvragée du Jardin des Retrouvailles à Lille (Nord), 1997.

(c) Laurence Baudelet. métropoles et leur périphérie mais il a aussi essaimé en milieu rural, en Occitanie comme en Bretagne. Selon un décompte réalisé en 2016 par le Réseau national des jardins partagés ${ }^{35}$ pour le Commissariat général à l'Égalité des territoires, il existait plus de 1000 jardins recensés dans les bases de données de ses correspondants présents dans neuf régions métropolitaines sur treize. Les territoires et départements d'outre-mer semblent être restés à l'écart de cette tendance globale bien que plusieurs projets aient vu le jour.

En mettant en avant la question du «partage » et de la " solidarité », du « lien social » et $\mathrm{du}$ « respect de l'environnement ", le Jardin dans tous ses états entendait répondre à de nouvelles attentes sociales et montrer que les jardins partagés pouvaient être un outil de réponse autant qu'une nouvelle forme d'utopie (fig. 5). 
Figure 5

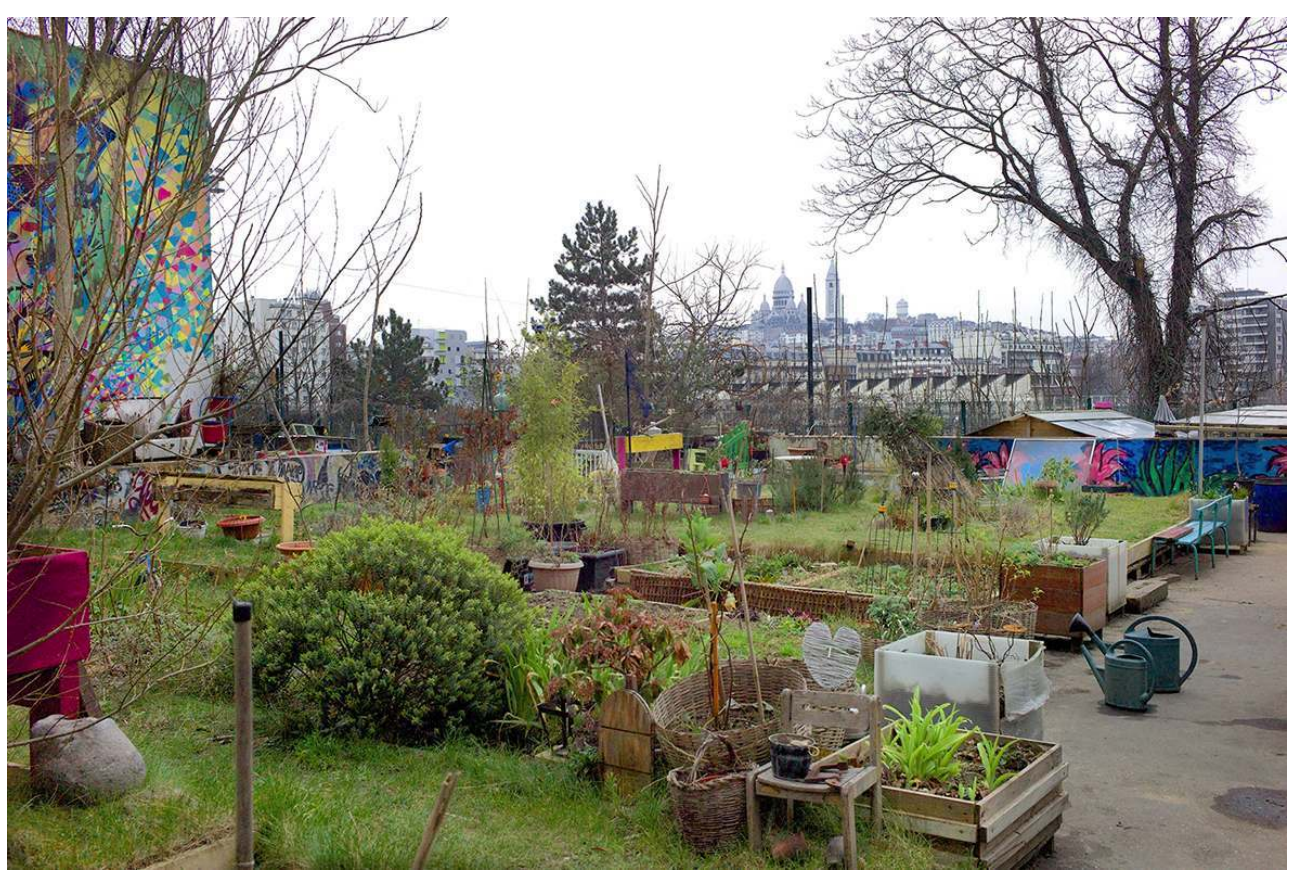

Ecobox, Paris 18e.

(c) Pierre-Emmanuel WECK, 2013.

\section{NOTES}

1. - BRAUDEL, Fernand. «Histoire et Sciences sociales : la longue durée ». Annales. Économies, Sociétés, Civilisations, 1958, n¹3-4, p. 725-753. Voir sur le site de Persée : https://www.persee.fr/ doc/ahess_0395-2649_1958_num_13_4_2781 [consulté le 03/10/2018].

2. - Réseau national des jardins partagés. Charte du jardin dans tous ses états - La terre en partage, 1997. Voir sur le site: http://jardins-partages.org/IMG/pdf/JTSE-leger.pdf [consulté le 03/10/2018].

3. - Le Jardin du cheminot est devenu Jardinot en 2005. Cette œuvre sociale gère des groupes de jardins qui étaient réservés aux employés du chemin de fer jusqu'en 2005. L'association s'est depuis ouverte à tous. Voir le site: http://www.jardins-familiaux.asso.fr/ [consulté le 03/10/2018].

4. - Voir le site : https://www.jardiniersdefrance.com/ [consulté le 29/11/2018].

5. - Voir le site : https://www.association-les-jardins-daujourdhui.asso-web.com/ [consulté le 29/11/2018].

6. - Voir le site : http://www.reseaucocagne.asso.fr/ [consulté le 29/11/2018].

7. - Voir le site : http://www.padesautoproduction.net/ [consulté le 29/11/2018].

8. - Voir le site : http://reseauecoleetnature.org/ [consulté le 29/11/2018].

9. - Voir le site : http://www.terrevivante.org/ [consulté le 29/11/2018]. 
10. - En 1997, cette association s'appelait Fédération des jardins familiaux. Elle a changé de nom en 2006.

11. - CABEDOCE, Béatrice, PIERSON, Philippe (dir.). Cent ans d'histoire des jardins ouvriers, 1896-1996. Grane : Créaphis, 1996.

12. - DUBOST, Françoise, LIZET, Bernadette. «La nature dans la cité, de l'hygiénisme au développement durable ». Communications, 2003, n 74 , « Bienfaisante nature », p. 5-18. Voir sur le site de Persée : https://www.persee.fr/doc/comm_0588-8018_2003_num_74_1_2125 [consulté le 03/10/2018].

13. - LARBEY, Vincent. Jardins et jardiniers: les pieds dans la terre, la tête dans les nuages. Une anthropologie du potager. Sociologie. Montpellier : Université Montpellier 3, 2013.

14. - HESSE, Philippe-Jean, LE CROM, Jean-Pierre (dir.). La Protection sociale sous le régime de Vichy. Rennes : PUR, 2001, p. 183-236; voir le site : http://books.openedition.org/pur/16002 [consulté le 03/10/2018].

15. - DUBOST, Françoise, LIZET, Bernadette. Art. cit.

16. - BOUKHARAEVA, Louiza, MARLOIE, Marcel. «Des sols agricoles au service de la résilience urbaine : réflexions à partir du cas de la Russie ». Espaces et sociétés, 2011/4, nº147, p. 135-153; voir le site: https://www.cairn.info/revue-espaces-et-societes-2011-4-p-135.htm [consulté le 03/10/2018].

17. - Voir note 8.

18. - Voir le site : http://www.terrevivante.org/11-le-magazine.htm [consulté le 29/11/2018].

19. - Loi Labbé : Loi $n^{\circ} 2014-110$ du 06/02/2014 visant à mieux encadrer l'utilisation des produits phytosanitaires sur le territoire national.

20. - Réseau national des Jardins partagés. Charte de la terre en partage, 1997 ; voir sur le site : http://jardins-partages.org/IMG/pdf/JTSE-leger.pdf [consulté le 03/10/2018].

21. - Plan Écophyto 2008-2018; voir le site: http://agriculture.gouv.fr/le-plan-ecophyto-pourreduire-lutilisation-des-produits-phytosanitaires-en-france [consulté le 03/10/2018].

22. - Rencontres politiques organisées à Paris en septembre et décembre 2007 pour élaborer des mesures en matière d'environnement.

23. - BASSET, Frédérique, BAUDELET, Laurence, LE ROY, Alice. Jardins partagés : utopie, écologie, conseils pratiques. Mens : Terre Vivante, 2008.

24. - BAUDRY, Sandrine. Cultiver son jardin, s'inscrire dans la ville: approche anthropologique des community gardens de New York City. Thèse de doctorat, études anglophones. Université ParisDiderot - Paris VII : 2010. Voir sur le site: https://tel.archives-ouvertes.fr/tel-00962486/ document [consulté le 03/10/2018].

25. - Association des Jardins ouverts et néanmoins clôturés ; voir le site : www.ajonc.org [consulté le $03 / 10 / 2018]$.

26. - Green Guerillas ; voir le site : http://www.greenguerillas.org/ [consulté le 20/04/2018].

27. - BARTHOLOMEW, Mel. Square foot gardening, a new way to garden in less space with less work. Emmaus (Pennsylvanie) : Rodale Press, 2005 (rééd.).

28. - LANZA, Patricia. Lasagna gardening. Emmaus (Pennsylvanie) : Rodale Press, 1998.

29. - Green Thumb; voir le site : https://greenthumb.nycgovparks.org/ [consulté le 20/04/2018].

30. - Les politiques d'insertion 1980-2009; voir le site: http://www.vie-publique.fr/politiquespubliques/politiques-insertion/chronologie/ [consulté le 03/10/2018].

31. - TABARLY, Sylviane. «Les étapes de la politique de la ville en France». Géoconfluences. Ressources de géographie pour les enseignants, 2005 ; voir le site : http://geoconfluences.enslyon.fr/doc/territ/FranceMut/FranceMutDoc6.htm [consulté le 03/10/2018].

32. - Le passe-jardins; voir le site: https://www.lepassejardins.fr/boite-a-outils-31-fichespratiques [consulté le 03/10/2018].

33. - Proposition de loi relative aux jardins collectifs, 2003 ; voir sur le site : http://www.senat.fr/leg/ tas03-02.html [consulté le 03/10/2018]. 
34. - GENDRON, Corinne, GAGNON, Christiane. «Développement durable et économie sociale : convergences et articulations ». Les cahiers de la CRSDD, collection recherche, 2011, nº 2, p. 3-16 ; voir sur le site : http://www.crsdd.uqam.ca/Pages/docs/02-20112.pdf [consulté le 03/10/2018]. 35. - Le Jardin dans tous ses états a pris le nom de Réseau national des jardins partagés en 2016.

\section{RÉSUMÉS}

Le mouvement français des jardins partagés émerge en 1997 avec la rédaction de la charte du Jardin dans tous ses états - La terre en partage et la constitution du réseau national $\mathrm{du}$ "Jardin dans tous ses états", appuyée par la Fondation de France. Le groupe initial est composé d'associations historiques représentant les jardins familiaux et de nouveaux militants du jardinage collectif qui croisent le champ de la lutte contre l'exclusion sociale avec celui de l'écologie. Ils sont fortement influencés par le modèle nord-américain des « community gardens » ou jardins communautaires, en particulier par les villes de Montréal et New York qui comptent déjà des milliers de jardiniers. Ils contribueront à la diffusion de ces expériences en Europe en invitant des associations pionnières comme Green Guerillas à témoigner de leurs réalisations. Le mouvement des jardins partagés s'est fait l'écho de revendications plurielles en phase avec les enjeux contemporains que sont le respect de l'environnement, l'aspiration à une démocratie participative et le besoin de créer des liens sociaux. Vingt ans se sont écoulés depuis l'ouverture du jardin des (Re)trouvailles, dans le quartier Moulins à Lille. En une génération, le mouvement s'est considérablement développé dans les grandes métropoles mais il a aussi essaimé en milieu rural, de l'Occitanie à la Bretagne et des Hauts de France à la Provence en passant par la région Rhône-alpine et le Grand Est. Cet article retrace son émergence, les acteurs qui l'ont constitué et le contexte qui l'a vu naître.

The French community gardening movement started in 1997 with the drawing up of a founding text on all forms of gardens and of a 'shared earth' charter, 'La Terre en partage'. A national network, called 'Le Jardin dans tous ses états', was set up with financial support from the 'Fondation de France', a national trust federating charities and philanthropic organisations. The first community garden, 'Le Jardin des (Re)trouvailles' was opened in Lille in its Moulins neighbourhood. Within the space of a generation, the movement has grown dramatically in large cities, but it has also developed in rural areas such as the Occitanie region, for example, and in Brittany. This article tells the story the emergence of this community gardening movement in France, its protagonists and the historical context of its birth.

\section{INDEX}

Mots-clés : jardin partagé, histoire contemporaine, monde occidental, action citoyenne, association, social et environnemental, espace urbain, périurbain, co-construction

Keywords : community garden, contemporary history, Western world, citizens' initiative, association, social and environmental, urban space, peri-urban, co-construction 


\section{AUTEUR}

\section{LAURENCE BAUDELET-STELMACHER}

Directrice - Graine de Jardins contact@grainedejardins.fr 